\title{
Cancer Patients Have Necessary Been COVID-19 Vaccinated
}

\section{Attapon Cheepsattayakorn ${ }^{1,2^{*}}$, Ruangrong Cheepsattayakorn ${ }^{3}$ and Porntep Siriwanarangsun}

${ }^{1}$ Faculty of Medicine, Western University, Pathumtani Province, Thailand.

${ }^{2} 10$ th Zonal Tuberculosis and Chest Disease Center, Chiang Mai, Thailand.

${ }^{3}$ Department of Pathology, Faculty of Medicine, Chiang Mai University, Chiang Mai, Thailand.

\author{
"Correspondence: \\ Attapon Cheepsattayakorn, 10th Zonal Tuberculosis and Chest \\ Disease Center, 143 Sridornchai Road Changklan Muang Chiang \\ Mai 50100, Thailand, Tel: 6653 140767; 6653 276364; Fax: 66 \\ 53 140773; 6653273590.
}

Received: 14 August 2021; Accepted: 20 September 2021

Citation: Cheepsattayakorn A, Cheepsattayakorn R, Siriwanarangsun P. Cancer Patients Have Necessary Been COVID-19 Vaccinated. Trends Int Med. 2021; 1(1): 1-4.

ABSTRACT
Hypothetically, mRNA-vaccine-encapsulated-small liposomes and lipid carriers may accumulate through
the permeation retention and enhanced effect in tumor tissues. The recommendation that have been suggested
administering COVID-19 vaccines one to two weeks prior to a chemotherapy dose by many key-professional
organizations has not been practical with COVID-19 administration schedules (for examples; two doses of mRNA-
1273 (Moderna) are recommended to be given 28 days apart, whereas two doses of BNT162b2 (Pfizer/BioNTech)
are given 21 days apart, efficacy > 94\%), variable chemotherapeutic regimens, and limited COVID-19 vaccination
slot availability, contributing to allowing the most rapid COVID-19 vaccination of these immunosuppressed
cancer patients and due to lacking COVID-19-vaccine safety and the information immunogenicity in the context
of immune-system-stimulated immunotherapies (for examples; immune checkpoint inhibitor (ICI) therapy) and
general exclusion of malignancy-diagnosed patients in the clinical trials of currently approved COVID-19 vaccines.

Keywords

COVID-19, Coronavirus Disease 2019, Vaccination, Cancer, Immunocompromised.

\section{Abbreviations}

AACR: American Association for Cancer Research's COVID-19 and cancer task force, ADE: Antibody-Dependent Enhancement, ASCO: American Society of Clinical Oncology, CAR: Chimeric Antigen Receptor, ESMO: European Society for Medical Oncology, ICI: Immune Checkpoint Inhibitor, IDSA: Infectious Disease Society of America, IgG: Immunoglobulin G, mRNA: messenger Ribonucleic Acid, NCCN: National Comprehensive Cancer Network, OR: Odds Ratio, SARS-CoV-2: Severe Acute Respiratory Syndrome-Coronavirus-type 2, TBI: Total Body Irradiation, TKIs: Tyrosine Kinase Inhibitors, USA: United States of America, US CDC: United States Centers for Disease Control and Prevention.

\section{Introduction}

In patients with cancer, SARS-CoV-2 (COVID-19) can contribute to increasing morbidity and mortality [1-3] and decreased survival was found in patients with hematological and intrathoracic malignancies, poor performance status, comorbidities, and increased age [3-5]. Patients with hematological malignancies who were treated with stem cell transplantation and antiCD-20 antibody demonstrated lower rates of seroconversion, compared to COVID-19-infected-cancer patients [6, 7]. Patients with hematological malignancies might have substantially compromised B-cell and T-cell responses [8]. These study results indicated that following COVID-19 vaccination, overall high seroconversion rates could be anticipated in cancer patients due to different mechanisms and degrees of immune suppression, such as cell therapies (particularly chimeric antigen receptor (CAR)-T cell), anti-CD-20 antibody (B-cell depleting) therapies, stem cell transplantation, immunosuppressive effects of corticosteroid treatment, and cytotoxic-chemotherapy-bone-marrow-suppressive effects in certain subgroups of cancer patients [9]. Currently, there are lacking data in cancer patients in protection following SARSCoV-2 (COVID-19) infection, reinfection by various SARS-CoV-2 (COVID-19) variants, or COVID-19 vaccination although mucosal 
surface antigens (e.g. IgA and protective T-cell responses) might be similarly important in protection from natural SARS-CoV-2 (COVID-19) infection [10]. The association of carcinogenesis with genomic information encoding vaccines, particularly with very-transient-intracellular-presence-COVID-19-mRNA vaccines is likely very low [11].

mRNA Vaccination in Patients with Anti-cancer Therapeutics Hypothetically, mRNA-vaccine-encapsulated-small liposomes and lipid carriers may accumulate through the permeation retention and enhanced effect in tumor tissues $[12,13]$. The recommendation that have been suggested administering COVID-19 vaccines one to two weeks prior to a chemotherapy dose by many keyprofessional organizations has not been practical with COVID-19 administration schedules (for examples; two doses of mRNA-1273 (Moderna) are recommended to be given 28 days apart, whereas two doses of BNT162b2 (Pfizer/BioNTech) are given 21 days apart, efficacy $>94 \%$ ), variable chemotherapeutic regimens, and limited COVID-19 vaccination slot availability, contributing to allowing the most rapid COVID-19 vaccination of these immunosuppressed cancer patients [14] and due to lacking COVID-19- vaccine safety and the information immunogenicity in the context of immunesystem-stimulated immunotherapies (for examples; immune checkpoint inhibitor (ICI) therapy) [15] and general exclusion of malignancy-diagnosed patients in the clinical trials of currently approved COVID-19 vaccines [16].

In convalescent-COVID-19 patients, neutralizing antibody, memory $\mathrm{B}$ and memory $\mathrm{T}$ cells specific to SARS-CoV-2 (COVID-19) have been identified after six months of infection [17-19] and both antibody production and memory CD4+ T-cells sustained several months after SARS-CoV-2 (COVID-19) infection in rapidly resolved-symptom individuals [20]. Humoral and cell-mediated immunity to SARS-CoV-2 (COVID-19) are the integrated highly-effective-durable-protective key [21]. Antibody-dependent enhancement (ADE) of disease, mediated by virus-binding antibodies and do not neutralize the virus takes two main virus-binding forms (one form in dengue virus infection by virus-binding antibody and the internalization of the antibodyvirus complex through interaction with Fc-gamma receptor into replicated macrophages; the other form, non-neutralizing antibodies mediate the formation of incited-inflammation-immune complex) has been proposed as a COVID-19 vaccine-design concern due to high levels of antibodies in in vitro observations and in patients with severe COVID-19 with SARS-CoV-2 (COVID-19) taken-up macrophages [22]. No compelling evidence of ADE from convalescent plasma has been found [23-25]. Theoretically, ADE is reduced by elicited-antibody-response-on-neutralizing-epitopeCOVID-19 vaccines [21].

Inability to integrate into host genome, delivery into cytoplasm, avoidance of anti-vector immunity, eliciting strong humoral and cellular immunity, avoidance of introduction of pathogen, and easier to mass-production are the advantages of mRNA vaccines, whereas the requirement of lipid nanoparticle for delivery, easy degradation, and freezing storage are their disadvantages [21].

\section{Previous Involved Studies}

In the USA, a study of 273,00 cancer-diagnosed patients (total of 73 million patients) that 16,570 patients were diagnosed of COVID-19 demonstrated the increased number of COVID-19-infected cancer patients with adjusted OR of 7 [26]. COVID-19 patients with recently diagnosed leukemia, non-Hodgkin lymphoma, and lung cancer were highest odds of COVID-19 infection with adjusted OR of $12.2,8.5$, and 7.7, respectively [26] and demonstrated the greater risk of mortality (14.9\%) among COVID-19-infectedcancer patients, compared to COVID-19 patients without cancer (5.3\%) and cancer patients without COVID-19 infection (4.0\%) [26], whereas hematological-malignancy-diagnosed patients had increased risk of mortality at least 2.5 times, compared to patients with other cancers (at least 1.2 times) [27].

Cancer patients with COVID-19 vaccination undergoing chemotherapy, with exception of during periods of intensive chemotherapy are expected to generate COVID-19-protective responses that are similar to inactivated influenza [28, 29], pneumococcal polysaccharide, and hepatitis subunit $[29,30]$ vaccinations. Cancer patients being treated with targeted therapies (tyrosine kinase inhibitors (TKIs) (erlotinib, imatinib, sunitinib, or monoclonal antibodies (trastuzumab, etc.)) are reasonably expected generating protective responses with COVID-19 vaccination [21]. Cancer patients on ICI therapy are expected to produce protective responses following COVID-19 vaccination due to low risk of immune-related adverse events (IRAEs) found in cancer patients with ICI therapy receiving influenza vaccination [21,31,32]. In some settings, delaying ICI treatment in cancer patients may be safe from a perspective of cancer treatment [33]. Practically, cancer patients treated with lymphodepleting or anti-B cell or antiCD19 therapy or plasma-cell-depleting therapy are recommended to receive vaccination or COVID-19 vaccination at least 6 months after therapies $[28,30]$.

Sometimes, they may be flexible to optimize the timing of COVID-19 vaccinations (for example; COVID-19 vaccination followed by anti-B cell therapy several weeks later) depending on the urgency and phase of a cancer treatment in a patient [21]. Cancer patients on radiation therapy, particularly total body irradiation (TBI) that usually is given to spine and total lymph nodes for bone marrow suppression prior to other rare situations or stem cell transplantation should be COVID-19 vaccinated to produce protective immunity responses [21]. Cancer patients receiving mRNA vaccines are not specifically anticipated safety concerns [21]. Generally, live vaccines, particularly live COVID-19 vaccines are not recommended in cancer patients ongoing cytotoxic, lymphodepleting, or targeted therapies [2830]. COVID-19 vaccination has been recommended after approval of BNT162b2 (Pfizer) vaccine in cancer patients by the American Association for Cancer Research's (AACR's) COVID-19 and cancer task force [34], American Society of Clinical Oncology (ASCO) [35], European Society for Medical Oncology (ESMO) [36], Infectious Disease Society of America (IDSA) [35], National Comprehensive Cancer Network (NCCN) COVID-19 vaccination advisory committee [21], Society of Immunotherapy of Cancer [37], 
and the United States Centers for Disease Control and Prevention (US CDC) [21]. COVID-19 vaccination is strongly recommended in cancer patients, including their caregivers, whereas exactly representative data are not available, benefits likely outweigh risks of COVID-19 [21]. Immunization or COVID-19 vaccination in cancer patients undergoing adoptive cell therapy or organ transplantation should be delayed at least 3 months to maximize vaccine efficacy $[21,38]$. In patients with breast cancer, screening examinations of transient lymphadenopathy from COVID-19 vaccination should be performed either before first dose or 4-6 weeks after the second dose of a COVID-19 vaccine $[39,40]$.

\section{Conclusion}

For continuation of the quality of oncological care, cancer patients on clinical trials should be prioritized for COVID-19 vaccination that do not affect the eligibility of the clinical trials.

\section{References}

1. Bakouny Z, Hawley JE, Choueiri TK, et al. CD8+ T cells contribute to survival in patients with COVID-19 and cancer: current challenges and perspectives. Cancer Cell 2020; 38: 629-646.

2. Kuderer NM, Choueiri TK, Shah DP, et al. Clinical impact of COVID-19 on patients with cancer (CCC19): a cohort study. Lancet. 2020; 395: 1907-1918.

3. Mehta V, Goel S, Kabarriti R, et al. Case fatality rate of cancer patients with COVID-19 in a New York hospital system. Cancer Discov. 2020; 10: 935-941.

4. Grivas P, Khaki AR, Wise-Draper TM, et al. Association of clinical factors and recent anticancer therapy with COVID-19 severity among patients with cancer: A report from the COVID-19 and Cancer Consortium. Ann Oncol 2021; 32: 787-800.

5. Robilotti EV, Babady NE, Mead PA, et al. Determinants of COVID-19 disease severity in patients with cancer. Nat Med. 2020; 26: 1218-1223.

6. Marra A, Generali D, Zagami P, et al. Seroconversion in patients with cancer and oncology health care workers infected by SARS-CoV-2. Ann Oncol. 2020; 32: 113-119.

7. ThakkarA, Pradhan K, Jindal S, et al. Patterns of seroconversion for SARS-CoV-2 IgG in patients with malignant disease and association with anticancer therapy. Nat Cancer. 2021; 2: 392399.

8. Chamilos G, Lionakis MS, Kontoyiannis DP. Are all patients with cancer at heightened risk for severe coronavirus disease 2019 (COVID-19) ? Clin Clinfect Dis. 2021; 72: 351-356.

9. Thakkar A, Gonzalez-Lugo JD, Goradia N, et al. Seroconversion rates following COVID-19 vaccination among patients with cancer. Cancer Cell. 2021; 39: 1-10.

10. Korompoki E, Gavriatopoulou M, Kontoyiannis DP. COVID-19 vaccines in patients with cancer-a welcome addition, but there is need for optimization. JAMA Oncology. 2021; 7: 1113-1114.

11. Górecki DC, Simons JP. The dangers of DNA vaccination. Nat Med 1999; 5: 126.

12. Kang CK, Kim HR, Song KH, et al. Cell-mediated immunogenicity of influenza vaccination in patients with cancer receiving immune checkpoint inhibitors. J Infect Dis. 2020; 222: 1902-1909.

13. Fanciullino R, Ciccolini J, Milano G. COVID-19 vaccine race: watch your step for cancer patients. British Journal of Cancer. 2021; 124: 860-861.

14. Desai A, Gainor JF, Hegde A, et al. COVID-19 vaccine guidance for patients with cancer participating in oncology Clinical Trials. Nat Rev Clin oncol. 2021; 18: 313-319.

15. Waissengrin B, Agbarya A, Safadi E, et al. Short-term safety on the BNT162b2 mRNA COVID-19 vaccine in patients with cancer treated with immune checkpoint inhibitors. Lancet Oncol. 2021; 22: 581-583.

16. Friese CR, Choueiri TK, Duma N, et al. Care without a compass: including patients with cancer in COVID-19 studies. Cancer Cell. 2021; 39: 895-896.

17. Wajnberg A, Amanat F, Firpo A, et al. Robust neutralizing antibodies to SARS-CoV-2 infection persist for months. Science 2020; 370: 1227-1230.

18. Dan JM, Mateus J, Kato Y, et al. Immunological memory to SARS-CoV-2 assessed for up to eight months after infection. Science. 2021; 371: eabf4063.

19. Rodda LB, Netland J, Shehata L, et al. Functional SARS-CoV2-specific immune memory persists after mild COVID-19. CCell. 2021; 184: 169-183.

20. Chen Y, Zuiani A, Fischinger S, et al. Quick COVID-19 healers ustain anti-SARS-CoV-2 antibody production. Cell. 2020; 183: 1496-1507e16.

21. Hwang JK, Zhang T, Wang AZ, et al. COVID-19 vaccines for patients with cancer : benefit likely outweight risks. Journal of Hematology and Oncology. 2021; 14: 38.

22. Lee WS, Wheatley AK, Kent SJ, et al. Antibody-dependent enhancement and SARS-CoV-2 vaccines and therapies. Nat Microbiol. 2020; 5: 1185-1191.

23. Libster R, Pérez Marc G, et al. Early high-titer plasma therapy to prevent severe COVID-19 in older adults. N Engl J Med. 2021; 384: 610-618.

24. Simonovich VA, Burgos Pratx LD, Scibona PB, et al. A randomized trial of convalescent plasma in COVID-19 severe pneumonia. N Engl J Med. 2021; 384: 619-629.

25. Agarwal A, Mukherjee A, Kumar G, C et al. Convalescent plasma in the management of moderate COVID-19 in adults in India: open label phase II multicentre randomized controlled trial (PLACID Trial). BMJ. 2020; 371: m3939.

26. Wang Q, Beiger NA, Xu R. Analyses of risk, racial disparity, and outcomes among US patients with cancer and COVID-19 infection. JAMA Oncol. 2020: 1-8.

27. Williamson EJ, Walker AJ, Bhaskaran K, et al. Factors associated with COVID-19-related death using open-safely. Nature. 2020; 1-17.

28. Rubin LG, Levin MJ, Ljungman P, et al. 2013 IDSA clinical practice guidelines for vaccination of the immunocopromised host. Clin Infect Dis 2013; 58: 44-100.

29. Mukulska M, Cesaro S, de Lavallade H, et al. Vaccination of patients with hematological malignancies who did not have transplantations: guidelines from the 2017 European 
Conference on infections in Leukaemia (ECIL 7). Lancet Infect Dis. 2019; 19: 188-199.

30. RigerCT, Liss B, Mellinghoff S, etal.Anti-infective vaccination strategies in patients with hematologic malignancies or solid tumors-gGidelines of the Infectious Diseases Working Party (AGIHO) of the German Society for Hematology and Medical Oncology (DGHO). Ann Oncol 2018; 29: 1354-1365.

31. Läubli H, Balmelli C, Kaufmann L, et al. Influenza vaccination of cancer patients during PD-1 blockade induces serological protection but may raise the risk for immune- related adverse events. J Immunother Cancer. 2018; 6: 40.

32. Keam B, Kang CK, Jun KI, et al. Immunogenicity of influenza vaccination in patients with cancer receiving immune checkpoint inhibitors. Clin Infect Dis. 2020; 71: 422-425.

33. Chen $\mathrm{G}, \mathrm{Wu} \mathrm{Q}$, Jiang $\mathrm{H}$, et al. Impact of treatment delay due to the pandemic of COVID-19 on the efficacy of immunotherapy in head and neck cancer patients. J Hematol Oncol. 2020; 13: 174.

34. Ribas A, Sengupta R, Locke T, et al. Priority COVID-19 vaccination for patients with cancer while vaccine supply limited. Cancer Discov. 2021; 11: 233-236.

35. Oncology ASoC. COVID-19 vaccine and patients with cancer. 2020. Available at: https://www.asco.org/ascocoronavirusresources/covid-19-patient-care-information/ covid-19-vaccine-patients-cancer (accessed on August 21, 2021).
36. Oncology ESfm. ESMO statements for vaccination against COVID-19 in patients with cancer. 2020. Available at: https:// www.esmo.org/covid-19-and-cancer/covid-19-vqccination (accessed on August 21, 2021).

37. Cancer Sflo. SITC statement on SARS-CoV-2 vaccination and cancer immunotherapy. 2020. Available at: https://www. sitcancer.org/aboutsitc/press-releases/2020/sitc- statementsars-cov-2-vaccination-cancer-immunotherapy (accessed on August 21, 2021).

38. National Comprehensive Cancer Network. Preliminary recommendations of the NCCN COVID-19 Vaccination Advisory Committee, 2021, Version 1.0 1/22/2021.NCCN. Available at :https://www.nccn.org/covid-19/pdf/COVID-19 (accessed on August 21, 2021).

39. Society of Breast Imaging Patients Care Delivery Committee. SBI Recommendations for the Management of Axillary Adenopathy in Patients with Recent COVID-19 Vaccination, 2021. Available at: https://www.sbi-online.org/Portals/O/ Position\%20Statements/2021/SBI-recommendations-formanaging-axillary-adenopathy-post-COVID- vaccination.pdf (accessed on August 21, 2021).

40. Edmonds CE, Zuckerman SP, Conant EF. Management of unilateral axillary lymphadenopathy detected on breast MRI in the era of coronavirus (COVID-19) vaccination. Am J Roentgenol. 2021. 